\title{
Interfacing MCNPX and McStas for simulation of neutron transport
}

Klinkby, Esben Bryndt; Lauritzen, Bent; Nonbøl, Erik; Willendrup, Peter Kjær; Filges, Uwe; WohImuther, Michael ; Gallmeier, Franz X.

Published in:

Nuclear Instruments and Methods in Physics Research Section A: Accelerators, Spectrometers, Detectors and Associated Equipment

Link to article, DOI:

10.1016/j.nima.2012.10.052

Publication date:

2013

Link back to DTU Orbit

Citation (APA):

Klinkby, E. B., Lauritzen, B., Nonbøl, E., Willendrup, P. K., Filges, U., Wohlmuther, M., \& Gallmeier, F. X. (2013). Interfacing MCNPX and McStas for simulation of neutron transport. Nuclear Instruments and Methods in Physics Research Section A: Accelerators, Spectrometers, Detectors and Associated Equipment, 700, 106-110. https://doi.org/10.1016/j.nima.2012.10.052

\section{General rights}

Copyright and moral rights for the publications made accessible in the public portal are retained by the authors and/or other copyright owners and it is a condition of accessing publications that users recognise and abide by the legal requirements associated with these rights.

- Users may download and print one copy of any publication from the public portal for the purpose of private study or research.

- You may not further distribute the material or use it for any profit-making activity or commercial gain

- You may freely distribute the URL identifying the publication in the public portal 


\title{
Interfacing MCNPX and McStas for simulation of neutron transport
}

\author{
Esben Klinkby ${ }^{* 1,3}$, Bent Lauritzen ${ }^{1,3}$, Erik Nonb $\varnothing 1^{1,3}$, Peter Kjær \\ Willendrup $^{2,3}$, Uwe Filges ${ }^{4,5}$, Michael Wohlmuther ${ }^{4,5}$, Franz X. Gallmeier ${ }^{6}$ \\ 1) DTU Nutech, Technical University of Denmark, DTU Risø Campus, \\ Frederiksborgvej 399, DK-4000 Roskilde \\ 2) DTU Physics, Technical University of Denmark, DTU Lyngby Campus, Anker \\ Engelunds Vej 1, DK-2800 Kgs. Lyngby \\ 3) ESS design update programme - Denmark \\ 4) Paul Scherrer Institute, CH-5232 Villigen PSI, Switzerland \\ 5) ESS design update programme - Switzerland \\ 6) Oak Ridge National Laboratory, Oak Ridge, Tennesee 37831, USA
}

\section{Abstract}

Simulations of target-moderator-reflector system at spallation sources are conventionally carried out using Monte Carlo codes such as MCNPX[1] or FLUKA $[2,3]$ whereas simulations of neutron transport from the moderator and the instrument response are performed by neutron ray tracing codes such as $\operatorname{McStas}[4,5,6,7]$. The coupling between the two simulation suites typically consists of providing analytical fits of MCNPX neutron spectra to McStas. This method is generally successful but has limitations, as it e.g. does not allow for re-entry of neutrons into the MCNPX regime. Previous work to resolve such shortcomings includes the introduction of McStas inspired supermirrors in MCNPX. In the present paper different approaches to interface MCNPX and McStas are presented and applied to a simple test case. The direct coupling between MCNPX and McStas allows for more accurate simulations of e.g. complex moderator geometries, backgrounds, interference between beam-lines as well as shielding requirements along the neutron guides.

Keywords: Neutron, Transport, Simulation, MCNPX, McStas, Interface

\footnotetext{
${ }^{*}$ Corresponding author
} 


\section{Introduction}

In the target-moderator-reflector system of a spallation source, neutrons are slowed down from being fast at the formation in the spallation target to thermal or cold in the beam extraction guides.

To model the interaction of a proton beam with a spallation target and to model the thermalization of the produced neutrons in moderators, the MCNPX code is a standard of its field [1]. Since mainly being developed for applications involving fast or thermal neutrons, however, the MCNPX code does lack in description of coherent scattering applicable to the cold/thermal range.

The transport of cold/thermal neutron through guides and optics and the simulation of scattering instruments on the other hand are well described using neutron ray-tracing codes such as McStas $[4,5,6,7]$. To bridge the gap between MCNPX and McStas, the approach has generally been to use analytical distributions fitted to MCNPX event spectra as input for the McStas simulation. This decoupled approach causes phase space information to be lost, and is limited by the fact that it does not allow the re-entry of McStas-simulated cold neutrons into the MCNPX regime. To estimate shielding requirements along a neutron guide or to calculate the gamma backgrounds relevant at neutron scattering instruments, it is necessary to apply MCNPX in calculating neutron absorption and gamma production ${ }^{1}$.

In order to resolve this issue, a more direct coupling between MCNPX and McStas is required. Below, various possibilities for such MCNPX-McStas coupling are described. Based on experience gained during implementation and tests of the interfaces, the feasibility and usefulness of the individual approaches are evaluated.

While the present paper is a pure computational study describing the various interfaces between MCNPX and McStas, experiments at the BOA beam-line ${ }^{2}$ are planned to validate against real measurements.

\section{Concepts for automated interfacing of MCNPX and McStas}

\subsection{Tally option - the present default}

This approach is based on fitting MCNPX neutron distributions e.g. at the moderator surface, allowing to model neutron states on a statistical basis. In short, a detailed MCNPX simulation of a target, reflector and moderator system of a given neutron facility is performed and the resulting neutron fluxes and energy spectra at the moderator surface are approximated by several Maxwellians. McStas then sample random neutron states from these distributions. A challenge faced when using this approach is to correctly describe the correlations between the parameters constituting a neutron state. For example, non-trivial

\footnotetext{
${ }^{1}$ Normally in a McStas guide simulation un-reflected neutrons are simply discarded.

${ }^{2}$ One of the beam-lines at the SINQ spallation source at the Paul Scherrer Institute (PSI), Switzerland.
} 
phase space correlations could exist e.g. between the neutron location and momentum at the moderator surface. Quantifying correlations is thus an important part of employing the Tally method.

The advantage of the Tally method, as seen from a user perspective is, that the time consuming MCNPX simulation step is decoupled from McStas and can be carried out once-and-for-all. This makes subsequent McStas simulations fast and therefore this method is very useful for e.g. instrument design.

\subsection{Ptrac option}

This approach utilises an intermediate step of event files, so that MCNPX at a given user-defined surface writes a file containing the state of the individual neutrons (position, momentum, time and Monte Carlo weight). An appropriate McStas interface exists to read in the neutron events. An advantage of using the Ptrac option with respect to the Tally approach is that all correlations between neutron state parameters are automatically conserved. Apart from the sizable intermediate files, a drawback by this approach is that the MCNPX code is unable to re-import data in the Ptrac format. I.e., this approach can only be a one way interface. Moreover the method is limited by the fact that MCNPX only allows particles crossing one surface to be written to file, and that the Ptrac option is unavailable under $\mathrm{MPI}^{3}$. For these reasons, relying on intermediate Ptrac files is inadequate as a general solution to the problem faced.

\subsection{Source Surface Write/Read (SSW/SSR) option}

$S S W / S S R$ is an MCNPX feature that allows to stop a simulation at a given surface, and restart it later. It is not intended to be used as a switch for external programs to be linked with an MCNPX simulation and the intermediate data files have undocumented MCNPX version dependent binary formats. A new interpreter has been developed, allowing McStas to run based on a $S S W / S S R$ file input, and to produce a $S S W / S S R$ output once the McStas simulation is complete. The main advantage of this approach compared to the Ptrac option is that MCNPX can run based on the $S S W / S S R$ input files. In this way one can first perform an MCNPX simulation of e.g. thermal neutron moderation. Once the neutrons enter the beam extraction region the neutron states are handed to the $S S W / S S R$ interface, and based on this a McStas simulation is carried out, e.g. involving mirrors and coherent scattering (which is not possible in MCNPX). The scattered and/or the non-scattered neutrons can then be handed back to MCNPX using the same interface.

The corresponding McStas components to read/write from/to the $S S W / S S R$ format are called: Virtual_MCNP_ss_input and Virtual_MCNP_ss_output, and are included as official McStas components starting from McStas version 2.0.

\footnotetext{
${ }^{3}$ MPI: Message Passing Interface, which is a method of parallelising computer processing. For additional information, see: http://www.mcs.anl.gov/research/projects/mpi/.
} 


\subsection{Compile option}

Closely resembling the above approach, the Compile option represents an even more direct coupling of the MCNPX and McStas codes. Rather than writing out intermediate files using the $S S W / S S R$ interface, the McStas and MCNPX codes are compiled together so that once a neutron arrives at a $\mathrm{Mc}$ Stas surface, a McStas simulation is launched from within MCNPX given a neutron state as input. After completion the updated neutron state is returned to MCNPX which proceeds the simulation. For an illustration see figure 1 . The Compile method is very flexible since all McStas functionalities are available from within MCNPX, but there are drawbacks: Firstly, the above relies on changes to the MCNPX code. Changes that would need to be repeated, if one would want to upgrade to later versions of MCNPX ${ }^{4}$. Secondly, there is a licensing issue at hand when merging the codes: McStas is licensed under GNU GPL v2 ${ }^{5}$, whereas MCNPX requires individual personal certification, something which many potential users may not be able to obtain. Thirdly, given the substantial difference in the CPU time consumption between typical MCNPX and McStas simulations (several orders of magnitude), there is an advantage in being able to separate them. If not, those e.g. designing/simulating neutron experiments at the end of the beam-line, would have to cope with very long simulation times since a full MCNPX simulation would have to be launched for each neutron. In many cases the lengthy simulations could be avoided with insignificant loss of precision if the McStas simulations were bootstrapped using the Tally, $S S W / S S R$ or Ptrac interface.

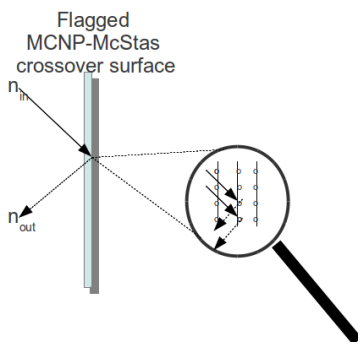

Figure 1: The McStas surface interface in MCNPX - illustration of coherent scattering from atomic lattice.

\subsection{Supermirror option}

Similar to the Compile option, the Supermirror option is based on modifying the MCNPX source code $[8,9]$. In this case, however, the idea is not to launch a McStas simulation from within MCNP, but rather to extend MCNPX, with

\footnotetext{
${ }^{4}$ Presently implemented in a development version of MCNPX 2.7. The cross release maintenance could be avoided if the changes were ported into the MCNPX development branch this will be attempted in the future.

${ }^{5} \mathrm{~A}$ special internal DTU and ESS project license for this usage was applied.
} 
functionality inspired from McStas. The first and most important shortcoming when using MCNPX for cold neutron applications is the lack of coherent scattering. Coherent scattering can be described as a neutron wave interacting with a lattice, while MCNPX only considers scattering on single particles. The process gives rise to wavelength-dependent reflection and can for the present purposes be well-approximated by the following expression [7]:

$$
\begin{array}{ll}
R=\frac{R_{0}}{2}\left(1-\tanh \frac{Q-m \cdot Q_{c}}{W}\right) \times\left(1-\alpha\left(Q-Q_{c}\right)\right) & \text { for } Q>Q_{c} \\
R=R_{0} & \text { otherwise }
\end{array}
$$

where $Q$ is the scattering vector, $Q_{c}$ is the critical scattering vector, $R_{0}$ is the low angle reflectivity constant, $W$ is the width of supermirror cut-off, $\alpha$ is the reflectivity slope, and $m$ is the $m$-value of the material. The wavelengthdependent reflectivity is depicted in figure 2 .

As for the Compile option, maintenance across MCNPX releases is problematic for the supermirror approach. Also McStas functionality other than supermirrors may need to be implemented, potentially requiring significant code development.

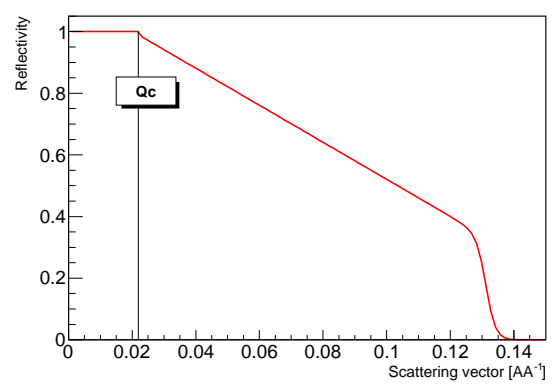

Figure 2: Supermirror reflectivity as a function of the scattering vector $Q$. The parameters used correspond to the McStas defaults: $Q_{c}=0.0219, m=2, W=0.003, R_{0}=0.99, \alpha=6.07$.

\section{Interface validation results}

In order to validate the performance of the interfaces presented above we consider a test scenario consisting of a source plane, that emits $10^{6} 20 \mathrm{meV}$ neutrons at a $45^{\circ}$ angle toward a mirror (i.e. a neutron guide), which then reflects the neutrons to the end wall for detection (surface current tally in MCNPX). The geometry is shown in figure 3 along with an example neutron trajectory. Focus below is put on the three interfaces presented here for the first time: $S S W / S S R$, Compile and Supermirror.

The SSW/SSR and Compile approaches are developed in MCNPX release 2.7 and to allow for direct comparison the existing Supermirror approach [8] was ported to the same release. 


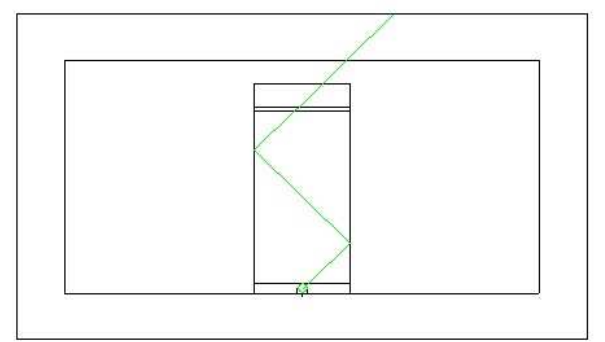

Figure 3: Geometry and example neutron simulation of the test setup used for MCNP-McStas interface validation. Illustrated using Vised[10].
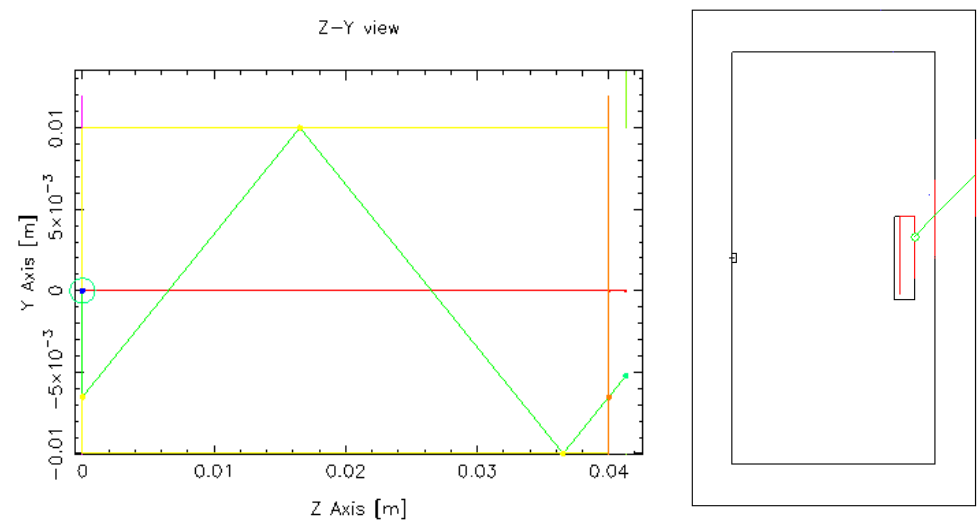

Figure 4: Example event of the SSW/SSR approach as visualized by McStas' mcdisplay and MCNPX's Vised[10].
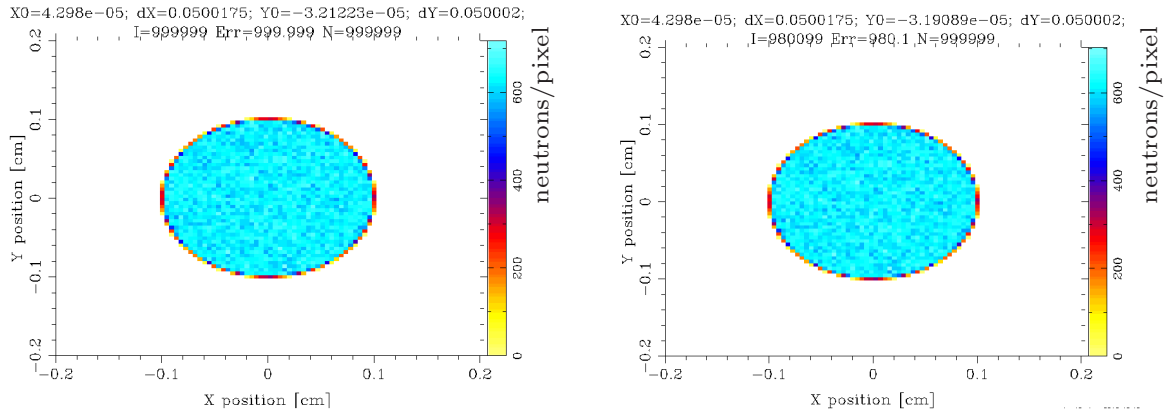

Figure 5: McStas PSD at the guide entrance (left) and exit (right). 

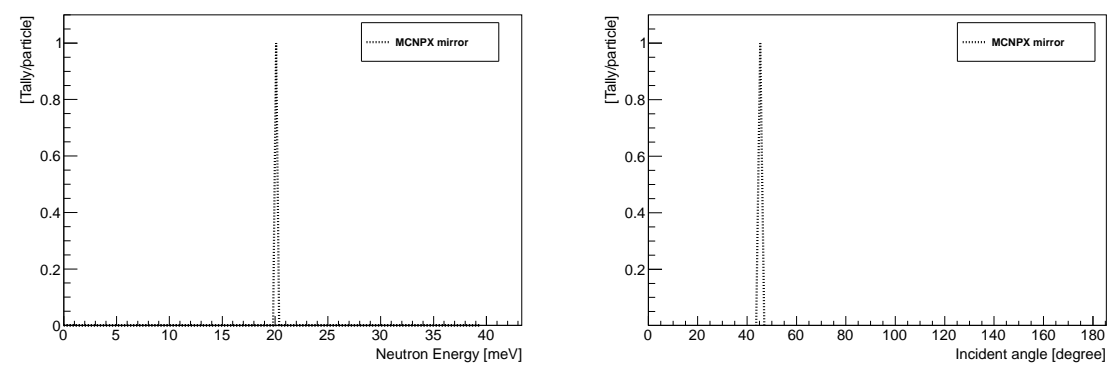

Figure 6: Spectrum and angular distributions at the guide exit using the MCNPX mirror. As expected both angle and energy are conserved.

We consider the MCNPX perfect specular mirror. Rather than choosing realistic settings for the guide (super)mirror, we choose the parameters of the guide such that nearly total reflection is expected, hereby enabling direct comparison against the MCNPX specular mirror. By also setting mass densities to zero, any bias due to lack of material effects in McStas is suppressed and thus, all methods should give identical neutron yield at the far end of the guide (apart from statistical fluctuations). To achieve this we set the following supermirror parameters: $R_{0}=0.99, Q c=20, m=2, W=0.003, \alpha=6.07$ (see Eq. 1) - note that $Q_{c}$ is a factor $\sim 1000$ above that of nickel (typical supermirror coating) to ensure maximum reflection, even at an angle of $45^{\circ}$.

To picture the process consider the $S S W / S S R$ interface. Immediately after the generation at the guide entrance, the neutrons are exported to McStas through the $S S W / S S R$ interface. Using the visualisation capabilities of McStas (mcdisplay) an example neutron is traced in figure 4(left). At the guide exit $(z=0.04 \mathrm{~m})$ it is returned to MCNPX and its final path through the tally surface is visualised using Vised[10] in figure 4(right). In figure 5 McStas Position Sensitive Detectors (PSD) placed at the guide entrance and exit of the guide show close to identical distributions, as expected given that the guide parameters are set to near total reflection. The result of a surface current tally at the far end of the guide (see figure 3 ) is shown in figure 6 .

\subsection{Cross Comparison}

After reentering in MCNPX, figure 7 shows the $S S W / S S R$, Compile and Supermirror performance in terms of spectrum and angular distributions at the guide exit (surface current tally). For comparison, also the MCNPX built-in mirror results are shown. The distributions agree very well both in terms of peak position and size (neutron yield).

Another check is presented in figure 8 which based on the same test geometry as figure 7 , but the vacuum in the guide is replaced by air ${ }^{6}$. Given that no

\footnotetext{
${ }^{6}$ Dry air according to standard composition provided by the MCNPX group.
} 
Surface current at guide exit
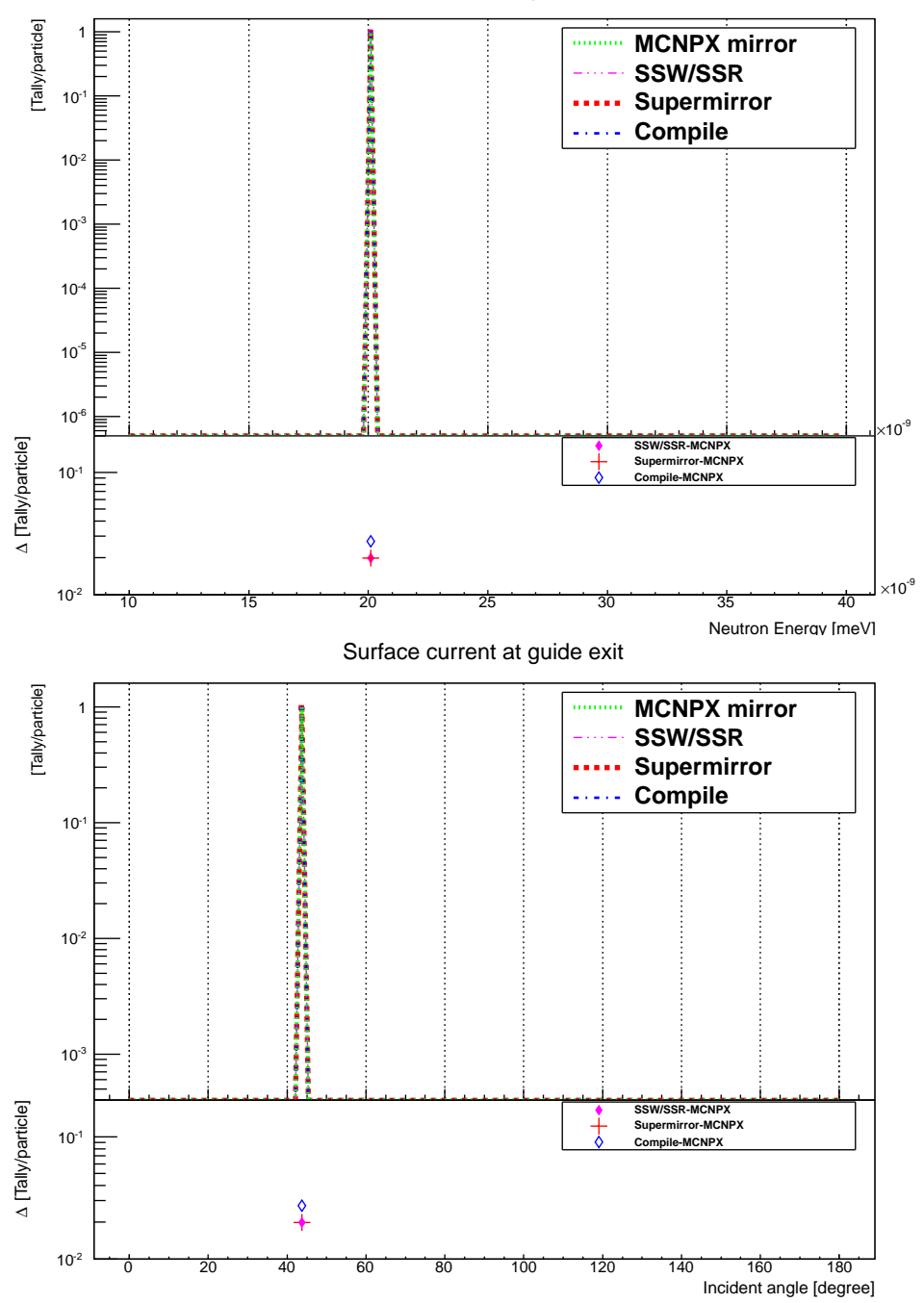

Figure 7: Spectrum (top) and incident angle comparison (bottom) of built-in specular MCNPX mirror, $S S W / S S R$, Compile and Supermirror approaches. The surface current tally is placed at the exit - see figure 3. The inserts below the main plots show the absolute differences of the various interface with respect to the built-in MCNPX specular mirror - the offset from zero is explained by the low angle reflectivity constant being different from unity. As expected all entries in the upper plot fall in the bin: $[19.6 ; 20.6] \mathrm{meV}$. Likewise, all entries in the lower plot fall in the bin covering the angular range: $[43.7 ; 45.4]^{\circ}$. 
attempt is made to simulate the effects of air in McStas, the non-zero bins in the histogram corresponding to $S S W / S S R$ may seem surprising at first glance. They are in fact due to neutrons back-scattering off air molecules after the guide. Thus the various interfaces are considered to be validated and the McStas plug5 ins to use them are made publicly available (under GNU licencing) via the 6 McStas homepage: www.mcstas.org.
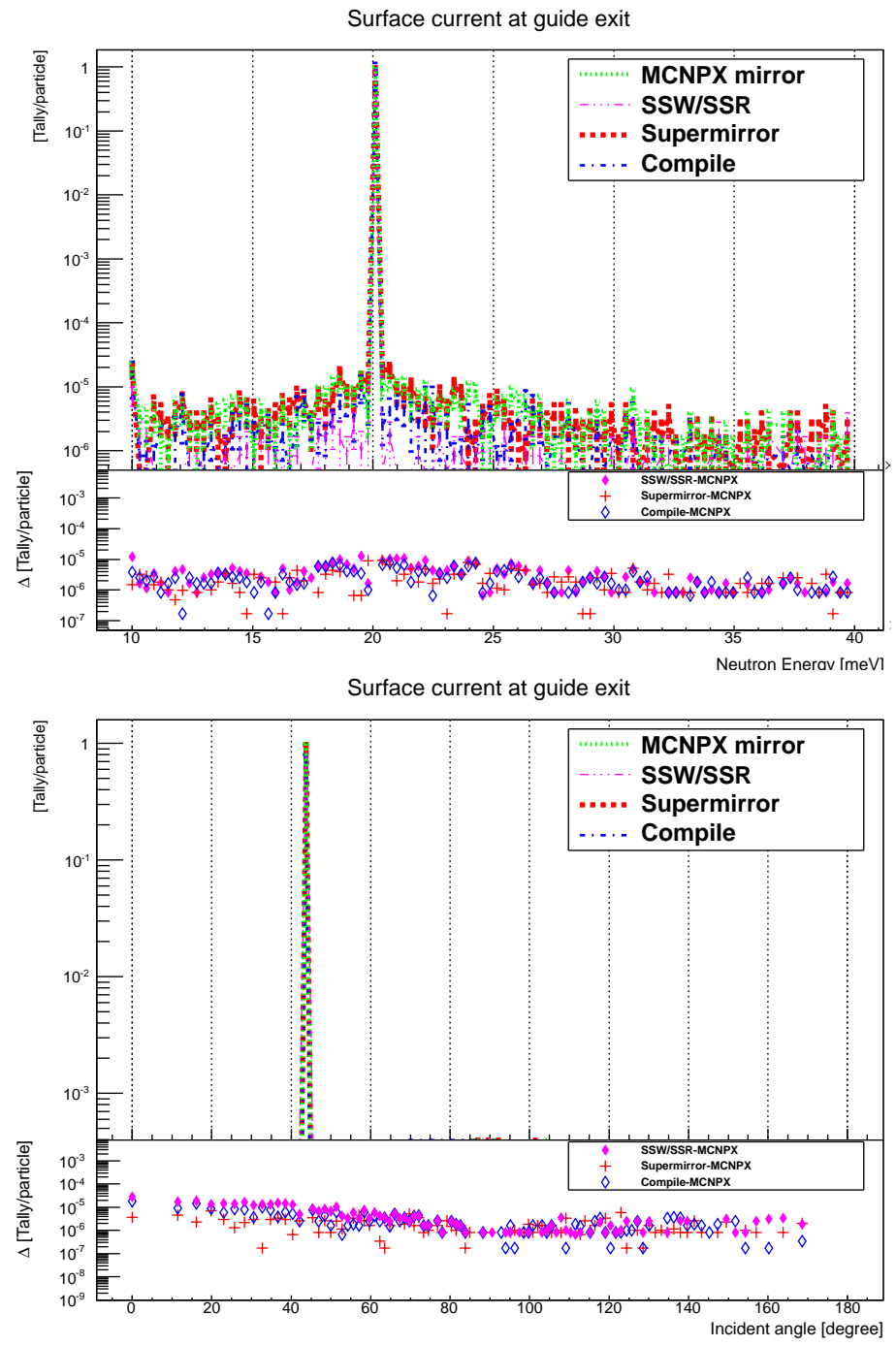

Figure 8: Logarithmic plots of spectra (top) and incident angle comparison (bottom) of builtin specular MCNPX mirror, SSW/SSR, Compile and Supermirror approaches using air-filled guide. The surface current tally is placed at the exit - see figure 3. The inserts below the main plots show the absolute differences of the various interface with respect to the built-in MCNPX specular mirror. 


\section{Prospects}

The introduction of supermirrors in MCNPX [8] has already proved very useful, and numerous simulations performed over the last years are based hereupon. Our goal is that the combination of MCNPX and McStas will extent the usability and become a new standard for detailed simulation of cold/thermal neutron moderators. Besides being directly applicable to the simulation of the target-moderator-reflector system of the spallation source, it will enable McStasbased descriptions of e.g. reflecting material and crystals to be included in the design and optimisation of advanced moderators. Examples include the recently proposed Si-crystal vanes [11] or nano-diamond coatings [12], which by using the directly coupled MCNPX-McStas interface could be simulated to a level beyond what is possible with the MCNPX or McStas codes alone. Also we foresee that the combination of MCNPX and McStas will enable more accurate calculation of photon production along neutron guides, and thus ultimately yield better shielding calculations. Finally, existing spallation sources have experienced problems with crosstalk between neutron guides. Given that e.g. the beam-lines at ESS are expected to be more closely spaced than at existing facilities, it is important already before the construction phase to start studying these effects, and we intend to do this using the coupled MCNPX McStas interface.

\section{References}

[1] L. S. Waters, G. W. McKinney, J. W. Durkee, M. L. Fensin, J. S. Hendricks, et al., The MCNPX Monte Carlo radiation transport code, AIP Conf.Proc. 896 (2007) 81-90. doi:10.1063/1.2720459.

[2] G. Battistoni, S. Muraro, P. R. Sala, F. Cerutti, A. Ferrari, et al., The FLUKA code: Description and benchmarking, AIP Conf.Proc. 896 (2007) 31-49. doi:10.1063/1.2720455.

[3] A. Ferrari, P. R. Sala, A. Fasso, J. Ranft, FLUKA: A multi-particle transport code (Program version 2005).

[4] K. Lefmann, K. Nielsen, McStas, a General Software Package for Neutron Ray-tracing Simulations, Neutron News 10 (1999) 20.

[5] P. Willendrup, E. Farhi, K. Lefmann, McStas 1.7 a new version of the flexible Monte Carlo neutron scattering package, Physica B 350 (2004) E735.

[6] P. Willendrup, E. Knudsen, E. Farhi and K. Lefmann, User and Programmers Guide to the Neutron Ray-Tracing Package McStas, Version 1.12c, Ris $\varnothing-R-1416$ (rev.ed.)(EN) (2011).

[7] P. Willendrup, E. Knudsen, K. Lefmann and E. Farhi, Component Manual for the Neutron Ray-Tracing Package McStas, Version 1.12, Ris $\varnothing-$ R-1538(rev.ed.)(EN) (2011). 
[8] F. X. Gallmeier, M. Wohlmuther, U. Filges, D. Kiselev, G. Muhrer, Implementation of neutron mirror modeling capability into mcnpx and its demonstration in first applications, Nuclear Technology 168(3) (2009) 768772 .

[9] D. Baxtor, A. Crabtree, P. Ferguson, F. Gallmeier, E. Iverson, W. Lu, G. Muhrer, Spallation neutron source moderator overview. Presented at IAEA Advanced Moderator Workshop, Tsukuba, Japan, November 22-25, 2011.

[10] http://www.mcnpvised.com/.

10

[11] E. Iverson, Contribution 336, ICANS XX, March 4 - 9, 2012. Bariloche, Rio Negro, Argentina.

12 [12] V. Nesvizhevsky, Nuclear Instruments and Methods in Physics, A 595 (2008) 631. 